\title{
Active surveillance in Canadian men with low-grade prostate cancer
}

\author{
Octav Cristea MD, Luke T. Lavallée MD, Joshua Montroy BSc, Andrew StokI MD, Sonya Cnossen MSc, \\ Ranjeeta Mallick PhD, Dean Fergusson PhD, Franco Momoli PhD, Illias Cagiannos MD, \\ Christopher Morash MD, Rodney H. Breau MD
}

CMAJ Podcasts: author interview at https://soundcloud.com/cmajpodcasts/150832-res

\begin{abstract}
Background: Recent guidelines recommend against routine screening for prostate cancer, partly because of the risks associated with overtreatment of clinically indolent tumours. We aimed to determine the proportion of patients whose low-grade prostate cancer was managed by active surveillance instead of immediate treatment.

Methods: We reviewed data for patients who were referred to the Ottawa regional Prostate Cancer Assessment Clinic with abnormal results for prostate-specific antigen (PSA) or prostate examination between Apr. 1, 2008, and Jan. 31, 2013. Patients with subsequent biopsy-proven low-grade (Gleason score 6) cancer were included. Active surveillance was defined a priori as monitoring by means of PSA, digital rectal examination and repeat biopsies, with the potential for curative-intent treatment in the event of disease progression.
\end{abstract}

Results: Of 477 patients with low-grade cancer, active surveillance was used for 210
$(44.0 \%)$, and the annual proportion increased from $32 \%(11 / 34)$ in 2008 to $67 \%(20 / 30)$ in 2013. Factors associated with immediate treatment were palpable tumour, PSA density above $0.2 \mathrm{ng} / \mathrm{mL}^{2}$ and more than 2 positive biopsy cores. Factors associated with surveillance were age over 70 years and higher Charlson comorbidity index. Of 173 men who received immediate surgical treatment, 103 (59.5\%) had higher-grade or advanced-stage disease on final pathologic examination. Of the 210 men with active surveillance, 62 $(29.5 \%)$ received treatment within a median of 1.3 years, most commonly (52 [84\%]) because of upgrading of disease on the basis of surveillance biopsy.

Interpretation: Active surveillance has become the most common management strategy for men with low-grade prostate cancer at our regional diagnostic centre. Factors associated with immediate treatment reflected those that increase the risk of higher-grade tumours.
$\mathrm{P}$ rostate cancer is the most commonly diagnosed noncutaneous malignancy and the third leading cause of cancer-related death in Canadian men. ${ }^{1}$ Screening based on serum prostate-specific antigen (PSA) has been used with the aim of detecting prostate cancer at an early stage, thus increasing the likelihood of successful treatment. ${ }^{2}$ However, clinical practice guidelines recently published by the United States Preventive Services Task Force ${ }^{3}$ and the Canadian Task Force on Preventive Health Care ${ }^{4}$ have recommended against routine PSA screening, partly because of the risk of overdiagnosis and overtreatment of clinically indolent disease. Other groups, such as the American College of Physicians, ${ }^{5}$ have recommended a shared decision-making approach between patients and clinicians, recognizing the limitations and possible harms of screening.
Low-grade (Gleason score 6) prostate cancer is associated with a small chance of cancerrelated death. ${ }^{6}$ Since the introduction of PSA screening, low-grade tumours have constituted about $40 \%$ to $50 \%$ of newly diagnosed prostate cancers. ${ }^{7}$ The widespread diagnosis of low-grade prostate cancer has led to concerns that these patients are overtreated. ${ }^{3-5}$ In recent years, however, active surveillance has emerged as an alternative management strategy for men with lowgrade disease. ${ }^{8}$ Active surveillance consists of close observation via a regimen of periodic PSA measurements, digital rectal examinations and serial prostate biopsies, with the goal of offering curative therapy in the event of disease progression or reclassification. Evidence suggests that active surveillance for low-grade prostate cancer is a safe and feasible management option that is
Competing interests: For work unrelated to this article, Luke Lavallée has received grants and personal fees from Sanofi and personal fees from Ferring, and Illias Cagiannos has received personal fees from AbbVie and Ferring.

Christopher Morash has received fees for advisory board participation from AbbVie, Janssen, Astellas, Sanofi, Ferring and Bayer. No other competing interests were declared.

This article has been peer reviewed.

Accepted: Dec. 14, 2015

Online: Feb. 29, 2016

Correspondence to: Rodney Breau, rbreau@ ottawahospital.on.ca

CMAJ 2016. DOI:10.1503/ cmaj.150832 
well tolerated by patients ${ }^{9,10}$ and does not substantially compromise oncologic outcomes. ${ }^{11,12}$

Although a variety of selection criteria for active surveillance exist, most patients with a diagnosis of low-grade (Gleason score 6) cancer may be candidates. ${ }^{13,14}$ Current Cancer Care Ontario guidelines list active surveillance as the preferred management strategy for "low-risk (Gleason score $\leq 6)$ localized prostate cancer." ${ }^{15}$ Data substantiating the actual use of active surveillance in clinical practice are limited, however, and to our knowledge no such data exist from a Canadian setting.

Our primary objective was to determine the proportion of men with low-grade (Gleason score 6) prostate cancer receiving active surveillance as initial management. Our secondary objectives were to characterize the factors associated with immediate treatment and those associated with treatment after a period of active surveillance. We also sought to characterize pathologic outcomes in men with low-grade prostate cancer who underwent radical prostatectomy instead of active surveillance, to determine whether men are being appropriately selected for treatment.

\section{Methods}

We performed this retrospective cohort study at a regional prostate cancer diagnostic centre located within The Ottawa Hospital in Ottawa, Ontario. The study was approved by the Ottawa Health Science Network Research Ethics Board.

\section{Clinical setting}

We reviewed the records of a consecutive cohort of men referred to the Ottawa regional Prostate Cancer Assessment Clinic from Apr. 1, 2008, to Jan. 31, 2013. This referral centre can be accessed by any physician in the Champlain Local Health Integration Network, which serves a population of about 1.2 million. ${ }^{16}$ Referrals to the clinic can be made for assessment of abnormal PSA results, abnormal rectal examination findings or both. These measures represent subjective assessments of risk; as such, the thresholds for referral vary among primary care practitioners. All referrals to the clinic are prospectively entered into a database containing demographic, diagnostic and treatment information. The basic information in this database was used to generate a cohort of patients who, after referral to this clinic, underwent prostate biopsy and were given a diagnosis of lowgrade (Gleason score 6) prostate cancer. The decision to proceed with biopsy was made jointly by the assessing physician and the patient, with consideration of multiple clinical factors, including PSA level, patient age, PSA change over time and family history of prostate cancer.

\section{Data abstraction}

For all patients identified as having low-grade prostate cancer on the basis of biopsy results recorded in the database, manual chart review was used to verify the database information and to collect additional information. Patient and tumour characteristics that were collected included age, medical comorbidities, family history of prostate cancer, clinical stage of cancer at diagnosis, and pathologic details of prostate biopsy and prostatectomy specimens. Expert genitourinary pathologists practising at our centre interpreted all of the specimens. Three researchers (O.C., J.M., A.S.) performed the chart review, using a standardized abstraction template with prespecified fields and definitions. The senior abstractor (O.C.) examined a random subsample of records abstracted by the others to ensure consistency of data abstraction. Disagreements were discussed and resolved through consensus (which also involved R.H.B.).

\section{Definitions}

Active surveillance was defined a priori as monitoring by means of PSA, digital rectal examinaton and repeat biopsies, with the potential for curativeintent treatment in the event of disease progression. Watchful waiting was defined as a decision to forgo treatments with curative intent and to provide palliative treatments only in the event that the patient experienced symptom progression. The senior data abstractor reviewed all cases classified as watchful waiting to ensure that the documentation in the medical record met these criteria. Loss to follow-up, a decision to forgo treatment (watchful waiting) or a decision to delay treatment for other reasons was not considered active surveillance.

\section{Follow-up and outcomes}

For the active surveillance cohort, the time spent on active surveillance, the reasons for leaving active surveillance and the final pathologic results (for patients who eventually underwent radical prostatectomy) were abstracted from the medical records. Follow-up protocols for active surveillance varied among clinicians and patients, but generally consisted of PSA testing and rectal examination every 3 to 6 months. Patients usually underwent a second prostate biopsy within 1 year of the initial diagnosis and subsequent serial biopsies every 2 to 4 years, as well as for-cause biopsies as clinically indicated. Patients were considered lost to follow-up if more than 12 months had elapsed between their most recent follow-up visit and the time of data collection. Extraction of follow-up data was completed between July and October 2014.

The primary outcome was the proportion of 
patients with low-grade (Gleason score 6) prostate cancer whose disease was initially managed with active surveillance. Secondary outcomes were pathologic results for patients treated with immediate radical prostatectomy, the proportion of patients who received treatment after a period of active surveillance, the reasons for leaving active surveillance and the final pathologic results for patients treated with radical prostatectomy after a period of surveillance.

\section{Statistical analysis}

Baseline patient and disease characteristics were stratified according to receipt of immediate treatment or management with active surveillance. Univariable and multivariable log binomial regression was performed to assess the associations between patient and disease characteristics and the risk of immediate treatment. A relative risk greater than 1 indicated a higher risk of immediate treatment. Results were considered statistically significant if the $p$ value was less than or equal to 0.05 . Follow-up time for the active surveillance cohort was calculated by subtracting the biopsy date from the treatment date or, for those still on active surveillance, from the date of last follow-up. A Kaplan-Meier curve constructed with time-to- event analysis was used to compute time on active surveillance, censoring at last follow-up or death. All statistical calculations were performed with SAS software, version 9.3 (SAS Institute Inc.).

\section{Results}

During the study period, 477 patients were evaluated and received a diagnosis of low-grade (Gleason score 6) prostate cancer. A total of 210 patients $(44.0 \%)$ were managed with active surveillance, whereas $244(51.2 \%)$ received immediate treatment (Figure 1). Eighteen of the patients $(3.8 \%)$ were followed with watchful waiting, $3(0.6 \%)$ were lost to follow-up before a management decision, and $2(0.4 \%)$ remained undecided about management approach at the time of data extraction. Baseline characteristics are summarized in Table 1.

\section{Trends in active surveillance and predictors of immediate treatment}

The proportion of patients managed with active surveillance increased from $32 \%$ (11 of 34) in 2008 to $67 \%$ (20 of 30) in 2013 (see Appendix 1, available at www.cmaj.ca/lookup/suppl/doi:10.1503/ cmaj.150832/-/DC1). Univariable analysis showed

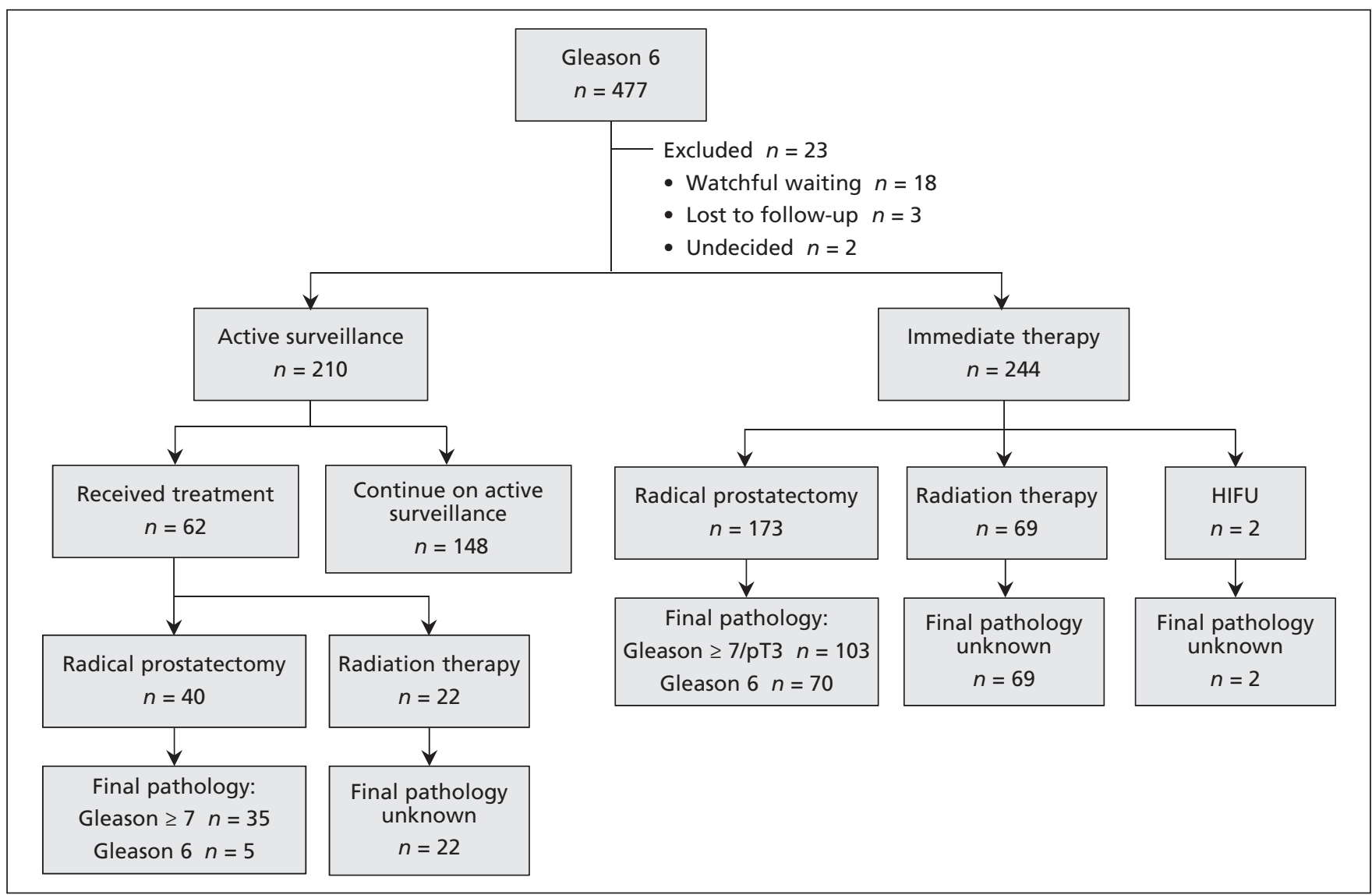

Figure 1: Summary of management decisions and pathologic outcomes. Gleason = Gleason score, HIFU = high-intensity focused ultrasound, pT3 = pathologic tumour stage 3 . 
that palpable tumours on digital rectal examination, PSA density greater than $0.2 \mathrm{ng} / \mathrm{mL}^{2}$, more than 2 positive biopsy cores and more than $50 \%$ cancer in any biopsy core were associated with immediate therapy; age older than 70 years, higher score on the Charlson comorbidity index and later year of biopsy were associated with active surveillance (Figure 2). All of these variables, with the exception of more than $50 \%$ cancer in any biopsy core, remained independently associated with management choice on multivariable analysis (Figure 3). Neither family history of prostate cancer nor serum PSA levels were significantly associated with the decision to manage with active surveillance.

\section{Pathologic outcomes for men undergoing immediate radical prostatectomy}

Of the 244 patients who received immediate treatment, $173(70.9 \%)$ underwent radical prostatectomy, $69(28.3 \%)$ underwent radiation therapy, and $2(0.8 \%)$ underwent high-intensity focused ultrasound. Of the 173 men who underwent radical prostatectomy, $103(59.5 \%)$ had upgrading to Gleason score of 7 or higher or reclassification to pathologic tumour stage 3 (i.e., pT3) on final pathologic examination.

\section{Experience of men on active surveillance}

Of the 210 patients who chose active surveillance, $62(29.5 \%)$ eventually received treatment: 22 $(35 \%)$ were treated with radiation and $40(65 \%)$ underwent radical prostatectomy. The median time from initial diagnosis to treatment was 1.3 (interquartile range [IQR] 0.9-1.8) years. Reasons for leaving active surveillance were reclassification to a higher risk state because of upgrading on repeat biopsy for 52 patients $(84 \%)$, patient preference or anxiety for 7 patients $(11 \%)$, a rapidly rising PSA for 2 patients $(3 \%)$ and increased number of biopsy cores containing cancer in 1 patient $(2 \%)$. Of the 22 patients treated with radiation, 21 had a Gleason score of 7 or higher and 1 had a Gleason score of 6

\begin{tabular}{|c|c|c|c|}
\hline \multirow[b]{2}{*}{ Characteristic } & \multicolumn{2}{|c|}{ Management approach; no. (\%) of patients* } & \multirow[b]{2}{*}{$p$ value } \\
\hline & $\begin{array}{l}\text { Active surveillance } \\
\qquad n=210\end{array}$ & $\begin{array}{l}\text { Immediate treatment } \\
\qquad n=244\end{array}$ & \\
\hline Age, yr, mean $\pm S D$ & $65.2 \pm 7.0$ & $60.9 \pm 7.1$ & $<0.001$ \\
\hline \multicolumn{4}{|l|}{ Charlson comorbidity index } \\
\hline 0 & $148(70.5)$ & $193(79.1)$ & 0.03 \\
\hline 1 & $39(18.6)$ & $39(16.0)$ & \\
\hline$\geq 2$ & $23(11.0)$ & $12 \quad(4.9)$ & \\
\hline \multicolumn{4}{|l|}{ Family history of prostate cancer } \\
\hline Yes & $39(18.6)$ & $63(25.8)$ & 0.1 \\
\hline No & $143(68.1)$ & $159(65.2)$ & \\
\hline Unknown & $28(13.3)$ & $22 \quad(9.0)$ & \\
\hline PSA at biopsy, ng/mL, mean \pm SD & $6.9 \pm 6.1$ & $6.4 \pm 4.4$ & 0.4 \\
\hline \multicolumn{4}{|l|}{ Clinical tumour (cT) stage } \\
\hline CT1 (not palpable) & $185(88.1)$ & $196(80.3)$ & 0.02 \\
\hline$\geq \mathrm{cT} 2$ (palpable) & $25(11.9)$ & $48(19.7)$ & \\
\hline PSA density, ng/mL ${ }^{2}$ & & $n=243$ & \\
\hline$\leq 0.2$ & $157(74.8)$ & $153(63.0)$ & 0.007 \\
\hline$>0.2$ & $53(25.2)$ & $90(37.0)$ & \\
\hline No. of positive biopsy cores & & $n=244$ & \\
\hline$\leq 2$ & $172(81.9)$ & $121(49.6)$ & $<0.001$ \\
\hline$>2$ & $38(18.1)$ & $123(50.4)$ & \\
\hline \multicolumn{4}{|l|}{ Highest \% cancer in a biopsy core } \\
\hline$\leq 50$ & $195(92.9)$ & $200(82.0)$ & $<0.001$ \\
\hline$>50$ & $15 \quad(7.1)$ & $44(18.0)$ & \\
\hline
\end{tabular}


on pretreatment biopsy. At 5-year follow-up, the estimated proportion of patients remaining on active surveillance was $59 \%$ (95\% confidence interval 49\%-68\%) (Figure 4). By last follow-up (at median 2.4 [IQR 1.3-3.3] yr), 180 patients $(85.7 \%)$ in the active surveillance cohort had

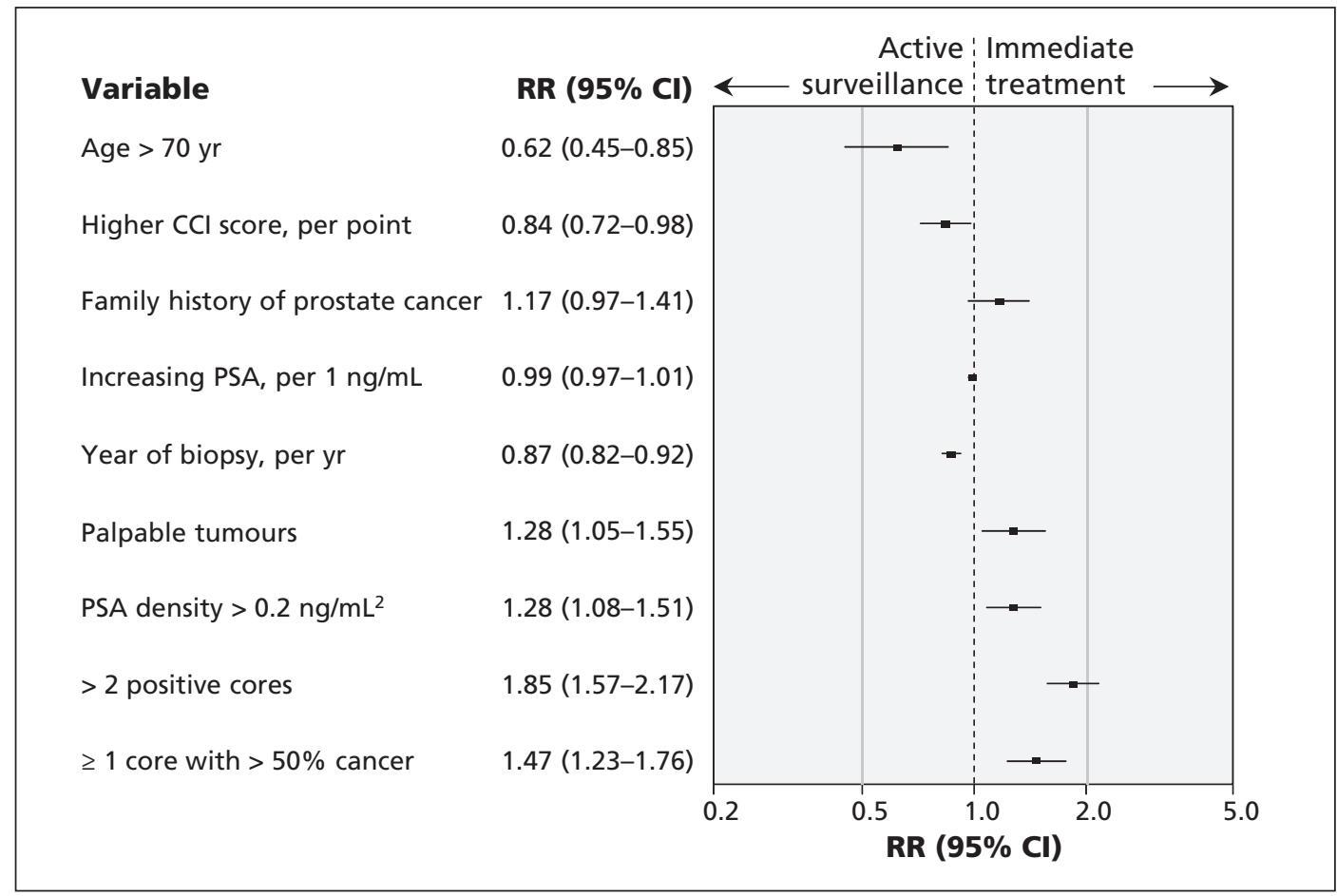

Figure 2: Univariable associations between baseline characteristics and use of immediate treatment following initial diagnosis of low-grade prostate cancer. $\mathrm{CCl}=$ Charlson comorbidity index, $\mathrm{Cl}=$ confidence interval, $\mathrm{PSA}=$ prostate-specific antigen, $\mathbf{R R}=$ relative risk. $\mathbf{R R}>1$ indicates higher risk of immediate treatment, and $R R<1$ indicates higher risk of active surveillance.

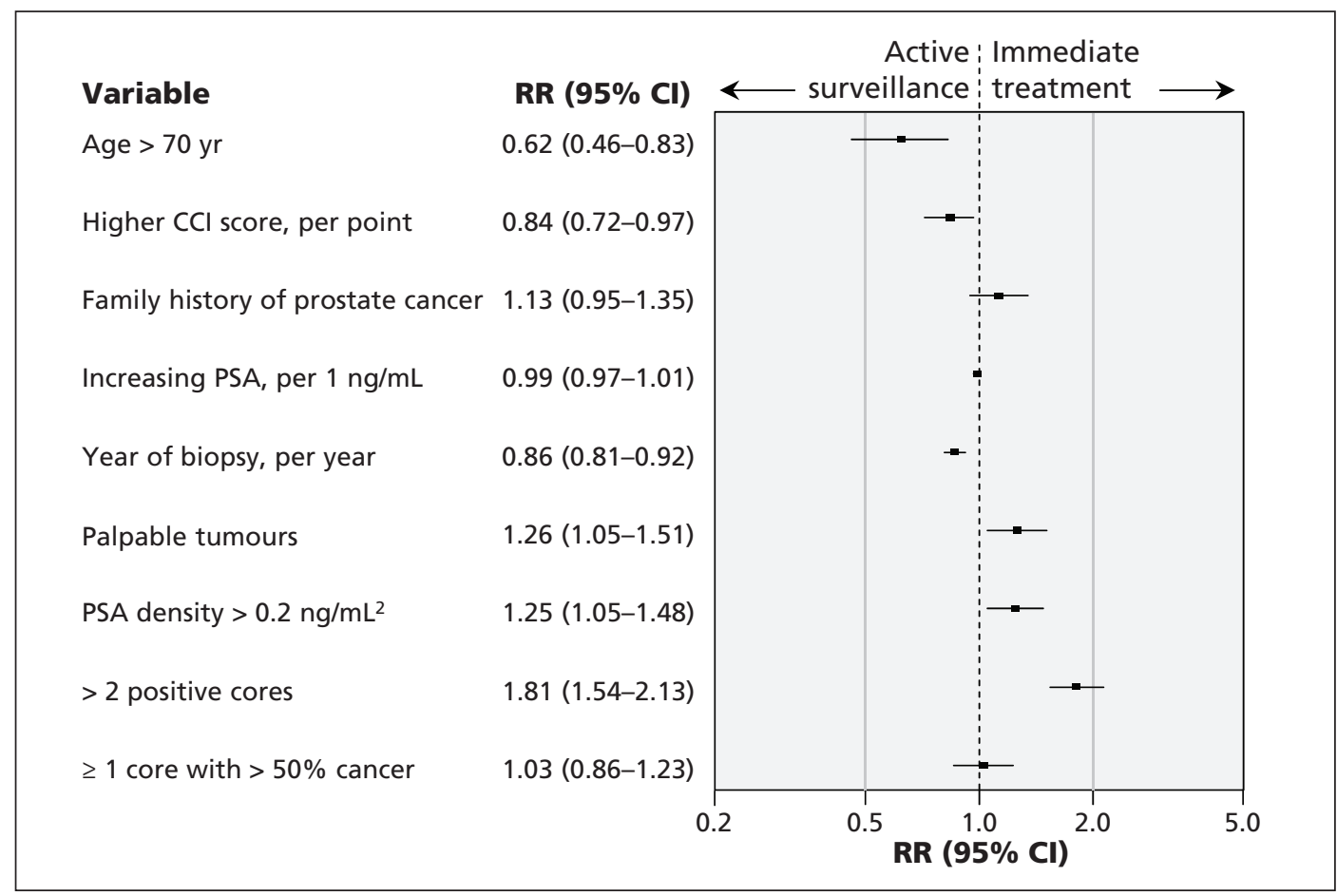

Figure 3: Multivariable associations between baseline characteristics and use of immediate treatment following initial diagnosis of low-grade prostate cancer. $\mathrm{CCl}=$ Charlson comorbidity index, $\mathrm{Cl}=$ confidence interval, PSA = prostate-specific antigen, $\mathbf{R R}=$ relative risk. $\mathbf{R R}>\mathbf{1}$ indicates higher risk of immediate treatment, and $R R<1$ indicates higher risk of active surveillance. 
undergone 1 or more repeat biopsies, and 54 $(25.7 \%)$ had undergone 2 or more biopsies. Twelve patients $(5.7 \%)$ were lost to follow-up, and 2 patients $(1.0 \%)$ died while on active surveillance, from unrelated causes.

\section{Pathologic outcomes for men with radical prostatectomy after active surveillance}

Forty of the 62 patients who received treatment after a period of active surveillance underwent radical prostatectomy. Of these, $35(88 \%)$ had an ultimate diagnosis of Gleason score 7 or higher cancer on final pathologic evaluation.

\section{Interpretation}

As of 2011, active surveillance became the most common management strategy for men with lowgrade prostate cancer at the Canadian regional diagnostic centre where this study was performed. Among the patients whose disease was managed with active surveillance, reasons for eventual treatment seemed to be based on a change in cancer characteristics and were rarely due solely to the patient's or physician's choice. These findings represent a substantial paradigm shift in the management of low-grade prostate cancer and may represent an important reduction in the potential harm associated with overtreatment of screeningdetected cancers.

In contrast to those who received active surveillance, patients who received immediate treatment in our region had clinical characteristics that are associated with more aggressive disease, such as higher PSA density, palpable tumours in the prostate and greater extent of tumour in prostate biopsy specimens. ${ }^{17,18}$ Indeed, most of the

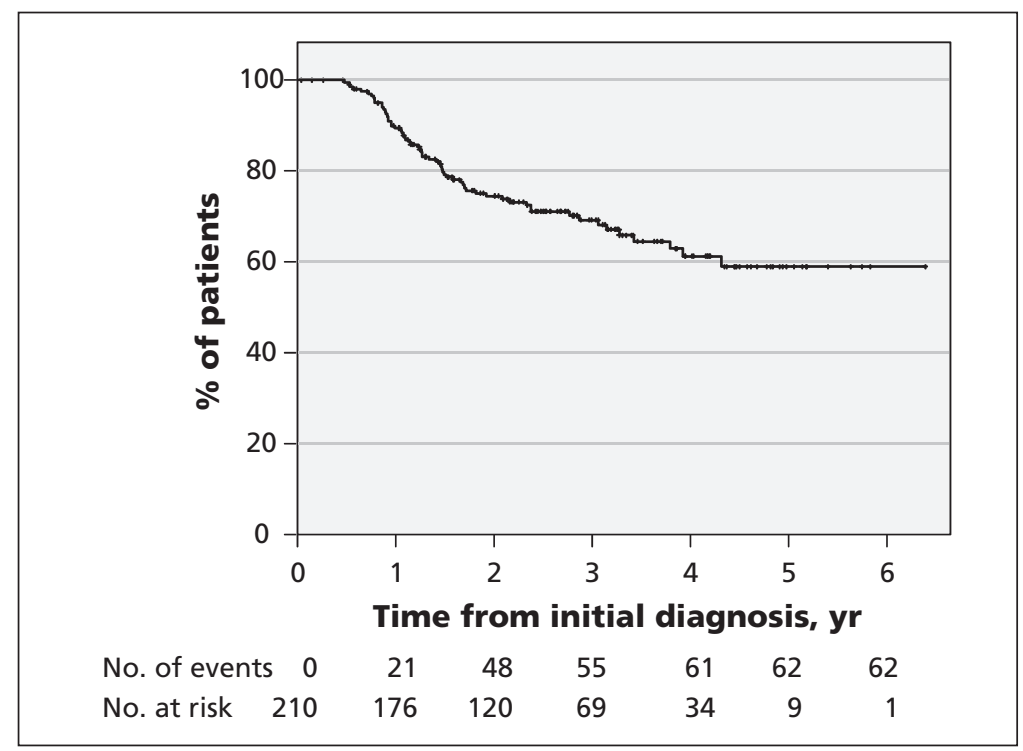

Figure 4: Kaplan-Meier curve for length of time on active surveillance since initial diagnosis. patients who underwent radical prostatectomy had upgrading or upstaging of their diagnosis when the prostatectomy specimen was examined. These data suggest that although active surveillance was commonly chosen, there was judicious use of definitive treatment for patients with an initial diagnosis of low-grade disease.

During the study period, 75 men underwent radical prostatectomy, either immediately upon diagnosis or after a period of surveillance, for what was ultimately determined to be localized lowgrade (Gleason score 6) disease on postprostatectomy pathologic examination. All of these patients may have received treatment for indolent disease. In addition, we do not have accurate pathologic characterization for a separate group of 72 individuals who underwent nonsurgical treatment based upon biopsy with a Gleason score of 6. If we assume that all of the nonsurgical patients had low-grade disease, the upper limit on overtreatment of low-grade prostate cancer was $147(30.8 \%)$ of the 477 patients.

Published data describing the proportion of low-risk patients with management by active surveillance are limited. Population-based data available from the US for patients treated between 2006 and 2009 showed no active treatment for $19.9 \%$ of men with low-risk disease. ${ }^{19}$ More contemporary cohorts from the US, Germany and Australia had surveillance rates of $19.4 \%$ to $39.0 \%$ for low-risk patients. ${ }^{20,21}$ Even in these studies, the true use of active surveillance may have been overestimated, as this specific management approach was not always differentiated from other forms of conservative management, such as watchful waiting. ${ }^{19,21}$ Regardless, it appears that active surveillance was used more frequently in our Canadian regional diagnostic centre than in other countries.

Consistent with other active surveillance cohorts, ${ }^{12,20,22-24}$ the majority of patients in this study remained on active surveillance during follow-up. In accordance with our results, some series have reported reclassification to highergrade cancer on repeat biopsy as the main reason for initiation of treatment after a period of active surveillance. ${ }^{23,24}$ Studies of other active surveillance cohorts, however, reported PSA doubling time as the most common indication for treatment, followed by reclassification to higher Gleason score. ${ }^{25,26}$ The difference in these latter series may, in part, reflect a longer follow-up time as well as a difference in the make-up of the active surveillance cohorts, with a proportion $(<20 \%)$ consisting of patients with intermediate-risk prostate cancer. In keeping with all of these studies, our results reinforce the concept that patient preference or anxiety does not appear to be the major factor driving progression to treatment. ${ }^{23-26}$ In the 
Canadian context, it seems that active surveillance is a feasible management approach that patients and physicians are willing to accept.

\section{Limitations}

This study had several limitations. The generalizability of the results may be limited, given that the data represent the experience of patients referred to a regional diagnostic centre, and practice patterns in other health networks may be different. Followup for this cohort was relatively short, and more patients in the active surveillance group would receive treatment with longer follow-up. Furthermore, we were unable to accurately characterize the true tumour grade and stage of patients who did not undergo radical prostatectomy. Finally, the pathologists who interpreted the prostatectomy specimens were not blinded to the patients' clinical history, and this lack of blinding may have biased the pathologic assessments.

\section{Conclusion}

Active surveillance has become the most commonly chosen management strategy for men with a diagnosis of low-grade prostate cancer in our regional diagnostic centre, regardless of PSA or clinical stage. Of the patients treated with immediate surgical therapy, final pathologic examination showed that most had intermediate- or high-risk disease. Our data suggest a growing uncoupling between the diagnosis of low-grade prostate cancer and immediate treatment. Although the cause of this trend is unknown, potential reasons include better knowledge of the natural history of low-grade prostate cancer and improved shared decisionmaking between clinicians and their patients.

\section{References}

1. Canadian Cancer Society Advisory Committee on Cancer Statistics. Canadian cancer statistics 2015. Toronto: Canadian Cancer Society; 2015.

2. Ilic D, Neuberger MM, Djulbegovic M. Screening for prostate cancer. Cochrane Database Syst Rev 2013;1:CD004720.

3. Moyer VA. Screening for prostate cancer: U.S. Preventive Services Task Force recommendation statement. Ann Intern Med 2012;157:120-34.

4. Bell N, Connor-Gorber S, Shane A, et al. Recommendations on screening for prostate cancer with the prostate-specific antigen test. CMAJ 2014;186:1225-34.

5. Qaseem A, Barry MJ, Denberg TD, et al. Screening for prostate cancer: a guidance statement from the Clinical Guidelines Committee of the American College of Physicians. Ann Intern Med 2013;158:761-9.

6. Albertsen PC, Hanley JA, Fine J. 20-year outcomes following conservative management of clinically localized prostate cancer. JAMA 2005;293:2095-101.

7. Klotz L, Emberton M. Management of low risk prostate cancer: active surveillance and focal therapy. Curr Opin Urol 2014;24:270-9.

8. Klotz L. Active surveillance for prostate cancer: overview and update. Curr Treat Options Oncol 2013;14:97-108.

9. Hayes JH, Ollendorf DA, Pearson SD, et al. Active surveillance compared with initial treatment for men with low-risk prostate cancer: a decision analysis. JAMA 2010;304:2373-80.

10. Wilcox CB, Gilbourd D, Louie-Johnsun M. Anxiety and health-related quality of life (HRQL) in patients undergoing active surveillance of prostate cancer in an Australian centre. BJU Int 2014;113 Suppl 2:64-8.

11. Satkunasivam R, Kulkarni GS, Zlotta AR, et al. Pathological, oncologic and functional outcomes of radical prostatectomy following active surveillance. J Urol 2013;190:91-5.

12. Klotz L, Vesprini D, Sethukavalan P, et al. Long-term follow-up of a large active surveillance cohort of patients with prostate cancer. J Clin Oncol 2015;33:272-7.

13. Klotz L. Active surveillance: patient selection. Curr Opin Urol 2013;23:239-44.

14. Reese AC, Landis P, Han M, et al. Expanded criteria to identify men eligible for active surveillance of low risk prostate cancer at Johns Hopkins: a preliminary analysis. J Urol 2013;190:2033-8.

15. Morash C, Tey R, Agbassi C, et al.; Active Surveillance Guideline Development Group. Active surveillance for the management of localized prostate cancer: guideline recommendations. Toronto: Cancer Care Ontario; 2015.

16. Population characteristics for Champlain Health Link Areas. Ottawa: Champlain LHIN; 2014. Available: www.champlainlhin. on.ca/ /media/sites/champlain/Accountability/Integration/HL\% 20Docs/201410ChHLAsPopCharEN.pdf?la=en (accessed 2015 May 26).

17. Vellekoop A, Loeb S, Folkvaljon Y, et al. Population based study of predictors of adverse pathology among candidates for active surveillance with Gleason 6 prostate cancer. $J$ Urol 2014;191:350-7.

18. El Hajj A, Ploussard G, de la Taille A, et al. Analysis of outcomes after radical prostatectomy in patients eligible for active surveillance (PRIAS). BJU Int 2013;111:53-9.

19. Hoffman KE, Niu J, Shen Y, et al. Physician variation in management of low-risk prostate cancer: a population-based cohort study. JAMA Intern Med 2014;174:1450-9.

20. Weerakoon M, Papa N, Lawrentschuk N, et al. The current use of active surveillance in an Australian cohort of men: a pattern of care analysis from the Victorian Prostate Cancer Registry. BJU Int 2015;115 Suppl 5:50-6.

21. Hager B, Kraywinkel K, Keck B, et al. Integrated prostate cancer centers might cause an overutilization of radiotherapy for low-risk prostate cancer: a comparison of treatment trends in the United States and Germany from 2004 to 2011. Radiother Oncol 2015;115:90-5.

22. Kates M, Tosoian JJ, Trock BJ, et al. Indications for intervention during active surveillance of prostate cancer: a comparison of the Johns Hopkins and Prostate Cancer Research International Active Surveillance (PRIAS) protocols. BJU Int 2015;115:216-22.

23. Dall'Era MA, Konety BR, Cowan JE, et al. Active surveillance for the management of prostate cancer in a contemporary cohort. Cancer 2008;112:2664-70.

24. Thomsen FB, Røder MA, Hvarness H, et al. Active surveillance can reduce overtreatment in patients with low-risk prostate cancer. Dan Med J 2013;60:A4575.

25. Klotz L. Active surveillance: the Canadian experience. Curr Opin Urol 2012;22:222-30.

26. Loeb S, Folkvaljon Y, Makarov DV, et al. Five-year nationwide follow-up study of active surveillance for prostate cancer. Eur Urol 2015;67:233-8.

Affiliations: Division of Urology (Cristea, Lavallée, Cagiannos, Morash, Breau), Department of Surgery, The Ottawa Hospital, Ottawa, Ont.; The Ottawa Hospital Research Institute (Montroy, Stokl, Cnossen, Mallick, Fergusson, Momoli, Breau), Ottawa, Ont.; Children's Hospital of Eastern Ontario Research Institute (Momoli), Ottawa, Ont.; University of Ottawa (Cristea, Lavallée, Momoli, Cagiannos, Morash, Breau), Ottawa, Ont.

Contributors: Octav Cristea, Dean Fergusson, Franco Momoli, Ilias Cagiannos, Christopher Morash and Rodney Breau were responsible for the study's conception and design. Octav Cristea, Luke Lavallée, Joshua Montroy, Andrew Stokl, Sonya Cnossen, Ranjeeta Mallick and Rodney Breau were responsible for data preparation and statistical analysis. Octav Cristea, Luke Lavallée and Rodney Breau drafted the manuscript. Joshua Montroy, Andrew Stokl, Sonya Cnossen, Ranjeeta Mattick, Dean Fergusson, Franco Momoli, Ilias Cagiannos and Christopher Morash critically revised the manuscript for important intellectual content. All of the authors approved the final version to be published and agreed to act as guarantors of the work.

Funding: No sponsorship or funding was obtained for this study. 